\title{
Hematopoietic cell transplantation in adults with acute myeloid leukemia: A review of the results from various nationwide registry studies in Japan
}

\author{
Yasuyuki Arai ${ }^{1}$, Takaaki Konuma², Masamitsu Yanada ${ }^{3}$ \\ ${ }^{1}$ Department of Hematology and Oncology, Graduate School of Medicine, Kyoto University, Kyoto, Japan, ${ }^{2}$ Division of \\ Molecular Therapy, The Advanced Clinical Research Center, The Institute of Medical Science, The University of Tokyo, \\ Tokyo, Japan, ${ }^{3}$ Department of Hematology and Cell Therapy, Aichi Cancer Center, Nagoya, Japan
}

\section{Abstract}

Hematopoietic cell transplantation (HCT) is an integral part of the treatment of acute myeloid leukemia (AML) . Although AML is the most common indication for allogeneic HCT, the relatively small number of patients and the highly complex nature of the procedure often render prospective studies on HCT unfeasible. Observational studies using registry data serve as important sources of information under such circumstances. Thus, the Japanese Society of Hematopoietic Cell Transplantation (JSHCT) has been conducting a nationwide survey of HCT since 1993 , and this registration program now covers $>99 \%$ of transplantation centers in Japan. A constant increase in the number of HCT procedures has been noted with recent annual registrations recording more than $>1,000$ new AML patients undergoing allogeneic or autologous HCT. Since its establishment in 2010, the Adult AML Working Group within the JSHCT has been using this registry data to complete several studies focusing on various aspects of $\mathrm{HCT}$ in adults with $\mathrm{AML}$, with particular attention paid to patient-, disease-, and transplantation-related factors. This article comprehensively reviews the results obtained from these studies and discusses the strengths and limitations of these kinds of registry-based studies.

Key words: acute myeloid leukemia, hematopoietic cell transplantation, allogeneic transplantation, autologous transplantation, registry study

Submitted December 22, 2020; Accepted February 10, 2021; Published online April 2, 2021; Issued online May 25, 2021

Correspondence: Masamitsu Yanada, Department of Hematology and Cell Therapy, Aichi Cancer Center, 1-1 Kanokoden, Chikusa-ku, Nagoya, 464-8681, Japan, E-mail: myanada@aichi-cc.jp

\section{Introduction}

Hematopoietic cell transplantation (HCT) is an effective treatment in patients with acute myeloid leukemia (AML). Allogeneic HCT has been widely used, while autologous HCT, which is still useful, is less prevalent because of concerns around post-transplant relapse, as well as the recently extended application of allogeneic HCT.

The Japanese Society of Hematopoietic Cell Transplantation (JSHCT) has been conducting a nationwide survey on HCT since 1993, in which HCTs performed at participating centers are registered with the JSHCT every year. Each participating center is required to register all consecutive HCTs, report core information for registered HCTs as of post-transplant day 100, and report follow-up information annually. This registration program currently covers nearly all of the $>300$ transplantation centers nationwide, with a penetration rate exceeding $99 \%{ }^{1}$. Since its establishment in 2010, the Adult AML Working Group at the JSHCT has been using this registry data to evaluate various aspects of HCT in adults with AML. The results of these studies are reviewed here.

\section{Overview}

Figure 1 shows the trend in the annual number of allogeneic and autologous HCTs in adults with AML reported in the Japanese transplantation registry. A constant increase in the annual number of allogeneic HCTs has been noted, with $>1,000$ adults with AML currently undergoing allogeneic HCT per year. This recent increase 


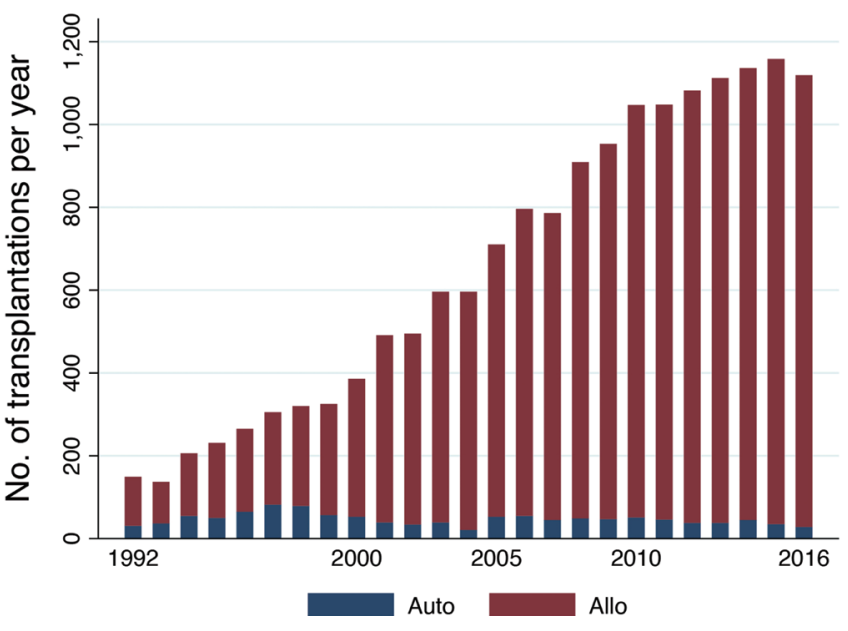

Figure 1. Trends in the annual number of allogeneic and autologous hematopoietic cell transplants in adults with acute myeloid leukemia reported to the Japanese transplantation registry

may be attributed to the growing use of unrelated HCT (Figure 2). The use of umbilical cord blood (UCB) has been steadily increasing, and UCB transplantation (UCBT) currently accounts for one-third of all allogeneic HCTs. Moreover, $>99 \%$ of the UCBTs were performed using a single UCB unit. The number of related HCTs has remained stable, while the use of peripheral blood stem cells (PBSCs) has now largely replaced that of bone marrow (BM). In cases of unrelated HCT, PBSC transplantation (PBSCT) still comprises only a very small percentage, which reflects the fact that unrelated PBSCT did not come into use in Japan until 2010. The 5-year probability of overall survival (OS) after allogeneic HCT was estimated to be $56 \%$ (95\% confidence interval $[\mathrm{CI}], 55 \%$ $57 \%$ ) for patients receiving a transplant while in CR, and $22 \%$ (95\% CI, 21\%-23\%) for those who were experiencing active disease. Various factors including age, sex, performance status (PS), disease status, cytogenetic risk, donor type, graft source, sex mismatch between the donor and the recipient, and year of transplantation were all shown to impact $\mathrm{OS}^{2}$.

The annual number of autologous HCTs peaked at 82 cases in 1997 and has remained at approximately 40 cases per year. The percentage of acute promyelocytic leukemia (APL) has sharply increased since the early 2000s and currently accounts for more than $70 \%$ of all autologous HCTs. The ratios for patients undergoing autologous HCT during the first CR (CR1) and the second CR (CR2) were $72 \%$ and $13 \%$ for non-APL patients and $23 \%$ and $65 \%$ for APL patients, respectively. The 5 -year OS probability after autologous HCT was estimated at $71 \%$ (95\% CI, 68\%-74\%) for patients undergoing transplant during CR and 31\% (95\% CI, 23\%-40\%) for those undergoing transplant while experiencing an

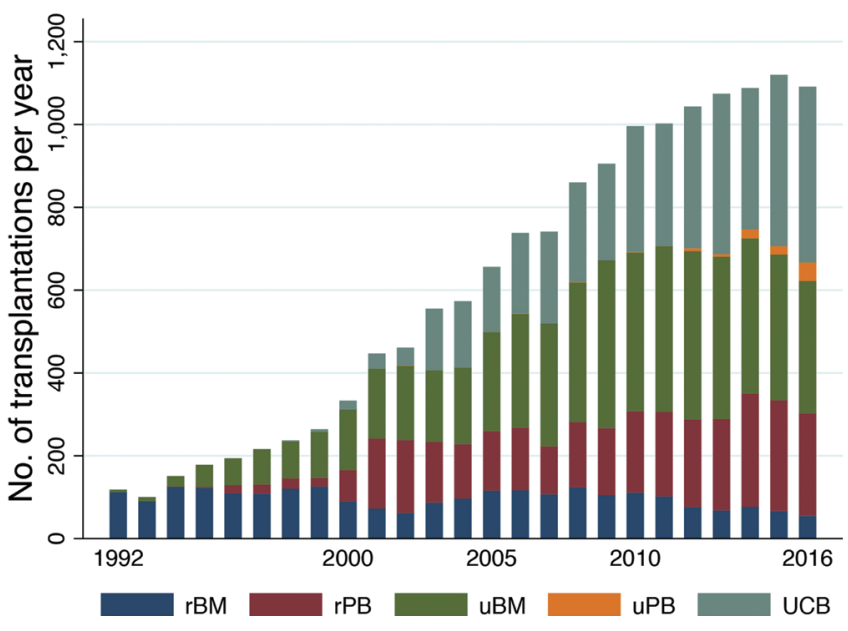

Figure 2. Trends in the annual number of allogeneic hematopoietic cell transplants in adults with acute myeloid leukemia according to donor type

rBM, related bone marrow; rPB, related peripheral blood stem cells; $\mathrm{uBM}$, unrelated bone marrow; uPB, unrelated peripheral blood stem cells; UCB, umbilical cord blood.

active disease state. Age, PS, disease status, cytogenetic risk, and year of transplantation were all shown to be closely associated with OS in non-APL patients, while sex and disease status were shown to be the most important contributing factors for APL patients ${ }^{3}$.

\section{Studies on patient-related factors}

The advent of reduced-intensity conditioning (RIC) has expanded the application of allogeneic HCT, resulting in a significant growth in the number of older patients undergoing allogeneic HCT. Given this change in practice, the prognostic impact of advanced age on allogeneic HCT needs to be reevaluated. In an analysis of patients aged $\geq 50$ years who underwent RIC allogeneic HCT, neither OS nor non-relapse mortality (NRM) differed for any of the age groups, 50-54, 55-59, 60-64, and $\geq 65$ years, evaluated. This study concluded that advanced age alone should not be considered a contraindication for allogeneic HCT and that closer attention should be paid to patients' organ function and the presence of comorbidities rather than their chronological age ${ }^{4}$. The outcomes of RIC allogeneic HCT in older patients has improved over time. A comparison of HCT data from 2008-2013 and that of 2000-2007 showed that OS for patients $>50$ years undergoing RIC allogeneic HCT was significantly better for the later cohort. This was found to be primarily attributable to reduced incidences of $\mathrm{NRM}^{5}$.

The existing NRM risk models for allogeneic HCT are not specific to patients with AML undergoing allogeneic HCT during CR1. Moreover, there is some debate about their predictive accuracy, with most experts agreeing that there is some room for improvement. Given this, our previous study developed a comprehensive system for pre- 
dicting NRM after allogeneic HCT during CR1 in AML. After randomly dividing 2,344 patients into a training or validation set, this study identified and scored five parameters, namely, age, sex, PS, HCT-specific comorbidity index (HCT-CI), and donor type, based on their impact on NRM in the patients in the training set. The new scoring system, named "NRM-J index", uses the sum of the assigned scores to stratify patients into four distinct risk groups $^{6}$. The application of the NRM-J index in the validation set demonstrated a better discriminative capacity than the European Society for Blood and Marrow Transplantation score ${ }^{7}$ and $\mathrm{HCT}-\mathrm{CI}^{8}$.

\section{Studies on disease-related factors}

Analyses focusing exclusively on AML patients provide valuable insights into disease-specific aspects during $\mathrm{HCT}$, and the use of large-scale registry data allows the evaluation of patients with rare AML subtypes. The studies presented in this section were designed to address issues related to cytogenetics, subtypes defined by the World Health Organization classification, extramedullary disease and disease status at transplantation.

\section{Cytogenetics}

A cohort of 7,812 patients was analyzed following their stratification as cytogenetically favorable-, intermediate-, and poor-risk groups as described by the National Comprehensive Cancer Network criteria used to evaluate the prognostic significance of cytogenetic risk in patients undergoing allogeneic HCT. Cytogenetic risk has been shown to have a major impact on post-transplant survival and relapse, and this effect was evident irrespective of donor type, disease status at the time of transplantation and conditioning intensity ${ }^{9}$.

The 11q23 chromosomal abnormalities in the KMT2A (formerly known as $M L L$ ) gene were detected in approximately $5 \%$ of adults with AML, and patients with these abnormalities generally have an unfavorable prognosis. The outcomes of 322 patients treated with allogeneic HCT were examined. The 3-year probabilities for OS, relapse, and NRM were $44 \%, 44 \%$, and $18 \%$, respectively, while the $11 \mathrm{q} 23$ fusion mutation was not predictive of OS. In CR patients, the presence of measurable residual disease at the time of transplantation proved to be predictive of higher relapse rates and worse $\mathrm{OS}^{10}$.

Additionally, several studies have evaluated the outcomes of allogeneic HCT in patients with specific cytogenetic abnormalities including trisomy $8^{11}, t(7 ; 11)(\mathrm{p} 15$; p15) ${ }^{12}$, and abnormalities in the short arm of chromosome $17^{13}$. These studies provided detailed information regarding factors affecting post-transplant outcomes for each cytogenetically distinct group and highlighted the importance of conducting allogeneic HCT during CR1 in these patients.

\section{Subtypes defined by the World Health Organiza- tion classification}

AML with myelodysplasia-related changes (AMLMRC) represents a specific category of AML, characterized by multilineage dysplasia (MLD), a history of myelodysplastic syndrome (MDS) or myelodysplastic/ myeloproliferative neoplasm (MDS/MPN), and MDSrelated cytogenetic abnormalities. Outcomes for these patients who had undergone allogeneic HCT were analyzed and compared to the outcomes in patients with AML not otherwise specified (AML-NOS). Patients with AML-MRC were shown to have worse OS than those with AML-NOS because of the significantly higher incidence of both relapse and NRM. Given the obvious prognostic impact of this disease subtype, patients with AMLMRC were further classified into three risk groups: a high-risk group including those with a monosomal karyotype, an intermediate-risk group including those with a complex karyotype and isolated $-5 / \mathrm{del}(5 \mathrm{q})$, and a lowrisk group made up of the remaining patients. The 3-year OS probabilities after allogeneic HCT were 14\%, 37\%, and $51 \%$ for high-, intermediate-, and low-risk patients, respectively ${ }^{14}$.

Given that the effectiveness of adding induction chemotherapy before allogeneic HCT for patients with AML-MLD remains uncertain, some patients proceed directly to allogeneic HCT without receiving induction chemotherapy. Recently a propensity score matching analysis was used to compare transplant outcomes for patients who did or did not receive induction chemotherapy before allogeneic HCT and evaluate the usefulness of this strategy. In this study, the CR status following induction chemotherapy was not considered because it was not known at the time of deciding whether to add induction chemotherapy. No differences in OS rates were observed between the two groups, and leukemia-related mortality was even lower in patients who did not receive induction therapy. These results provide some support for proceeding directly to allogeneic HCT in patients with AMLMLD $^{15}$.

The prognosis for patients with Philadelphia chromosome-negative MPNs is dismal once leukemic transformation occurs. We reported the results of allogeneic HCT in 39 patients comprising 21,11 , and 7 cases of essential thrombocythemia, primary myelofibrosis, and polycythemia vera, respectively, who had developed leukemic transformation. The disease status at the time of transplantation was non-CR in 32 patients. The 2-year OS, cumulative incidence of relapse, and NRM were $29 \%$, $34 \%$, and $34 \%$, respectively. No differences in outcomes were observed among these patients in terms of their underlying MPNs ${ }^{16}$. 
AML with minimal differentiation, which falls into the M0 category according to the French-American-British classification, has a poor prognosis. The analysis of 398 patients with AML with minimal differentiation undergoing allogeneic HCT showed that post-transplant outcomes were primarily dependent on disease status at transplantation as well as on cytogenetic risk for non-CR transplant patients. The 3-year OS rates were $60 \%$ and $59 \%$ for CR patients with intermediate or poor cytogenetics and $39 \%$ and $10 \%$ for non-CR patients with intermediate or poor cytogenetics, respectively ${ }^{17}$.

Acute erythroid and acute megakaryoblastic leukemia are infrequent subtypes of AML, with closely related cellular properties associated with both their morphology and gene expression profiles. Patient outcomes were analyzed for both subtypes following allogeneic HCT. OS was significantly worse in patients with acute megakaryoblastic leukemia than those with acute erythroid leukemia ( $17 \%$ and $35 \%$ at 5 years). This difference was due to a higher relapse rate in acute megakaryoblastic leukemia, suggesting the need to differentiate between these two subtypes in the HCT setting ${ }^{18}$.

Acute panmyelosis with myelofibrosis (APMF) is an extremely rare subtype of AML characterized by an acute onset of cytopenia, BM fibrosis and a dismal prognosis for chemotherapy alone. A total of 40 APMF patients undergoing allogeneic or syngeneic HCT were identified from the registry and subjected to detailed analyses. The disease status at HCT was CR1 in 13 patients, with the 27 other patients receiving transplant despite their active disease state. The 3-year OS and relapse rates were $24 \%$ and $59 \%$, respectively. Allogeneic HCT was shown to possess some curative potential in APMF despite a high relapse rate $^{19}$.

The prognosis of patients with biphenotypic acute leukemia (BAL) is usually poor because of their innate resistance to chemotherapy. We analyzed the treatment outcomes in 90 patients with BAL who had undergone allogeneic HCT. The probability of OS was $40 \%$ at 5 years, which was comparable to that of patients with AML and acute lymphoblastic leukemia (ALL). Multivariate analysis revealed no significant differences in OS, relapse, or NRM between BAL and AML or between BAL and ALL, which indicates that the benefits of allogeneic HCT may prevail despite the historically poor prognosis of $\mathrm{BAL}^{20}$.

\section{Extramedullary disease}

We studied whether a prior history of central nervous system (CNS) involvement adversely affected the outcome of allogeneic HCT. We found that the probabilities of OS, relapse, and NRM at 2 years were $51 \%, 30 \%$, and $15 \%$, respectively in 157 patients with a history of CNS involvement. This result was comparable with that of patients without a history of CNS involvement ${ }^{21}$.

In addition to the CNS, AML produces extramedullary involvement in a variety of tissues in the form of myeloid sarcoma and leukemia cutis. Moreover, the effect of extramedullary disease (EMD) on post-transplant outcomes was investigated. Regardless of the presence or absence of EMD, patients who received their transplant during CR showed no differences in relapse and OS rates. However, EMD was linked to higher relapse and reduced OS rates in non-CR patients when compared to those without $\mathrm{EMD}^{22}$.

\section{Disease status at transplantation}

Patients who achieve CR1 but experience relapse thereafter may still be salvaged with allogeneic HCT. Consequently, CR2 is the best time for allogeneic HCT in such patients. We examined the outcomes and prognostic factors for patients who received allogeneic HCT during CR2 and found that the 3-year OS, relapse, and NRM rates were $66 \%, 19 \%$, and $19 \%$, respectively. Multivariate analysis revealed that advanced age, poor cytogenetics, and shorter CR1 duration, especially when it was $<1$ year, were predictive of worse $\mathrm{OS}^{23}$.

The prognosis for patients with multiple relapses is poor. Analysis of patients with a history of multiple relapses before allogeneic HCT, revealed that allogeneic HCT still offers a potential cure for these patients. Furthermore, the outcomes differed significantly based on the disease status at the time of transplantation. Patients who underwent third or subsequent CR $(\mathrm{CR} 3+)$ had better OS than those transplanted during the second (REL2) or subsequent relapse (REL3 +; 48\%, 21\%, and $12 \%$ at 4 years, respectively) because of the reduced risk of post-transplant relapse $(23 \%, 57 \%$, and $52 \%$ at 4 years, respectively). Multivariate analysis revealed significantly lower relapse and overall mortality rates in patients with CR3 + and a significantly lower NRM for those in REL2. These results indicate the potential benefits and risks of reinduction attempts prior to transplantation and highlight the need for an individualized approach when determining whether to use reinduction therapy in this setting ${ }^{24}$.

\section{Studies on transplant-related factors}

Transplant-related factors are clinically important in the sense that physicians may choose between several available options. In this section, we discuss the studies designed to evaluate various issues around conditioning regimens and stem cell sources.

\section{Conditioning}

The combination of cyclophosphamide and total body irradiation $(\mathrm{CY} / \mathrm{TBI})$ is a widely used myeloablative conditioning (MAC) regimen. High-dose cytarabine 
(HDCA) may be incorporated into CY/TBI in the hope of intensifying its antileukemic effect, especially in patients with high-risk disease features. Two studies were conducted to evaluate the effectiveness of adding HDCA to CY/TBI（HDCA/CY/TBI). The first study analyzed patients undergoing UCBT following CY/TBI with or without HDCA. The addition of HDCA was found to reduce leukemia-related mortality without increasing NRM, leading to a significantly better OS in patients receiving $\mathrm{HDCA} / \mathrm{CY} / \mathrm{TBI}^{25}$. The second study analyzed patients undergoing allogeneic BM transplantation (BMT) or PBSCT. However, this study found that the addition of HDCA did not improve outcomes; leukemiarelated mortality did not decrease, and NRM was significantly higher. Consequently, OS was even worse for patients receiving HDCA/CY/TBI than for those receiving $\mathrm{CY} / \mathrm{TBI}$ alone ${ }^{26}$.

The choice between RIC and MAC is largely determined by the patient age and comorbidities. However, optimal conditioning intensity may depend on other factors including disease characteristics. An analysis of patients with poor-risk cytogenetics who underwent allogeneic HCT during CR1 revealed that MAC was associated with better OS than RIC because of the higher relapse incidence in patients receiving RIC. This study highlights the fact that there were better survival rates in patients who received the optimal conditioning intensity given their specific combination of disease features ${ }^{27}$.

There is less clinical data for RIC mediated allogeneic HCT in younger patients as MAC remains the conditioning of choice in these populations. This prompted us to conduct a comparative analysis of RIC and MAC in patients aged $<50$ years. This analysis revealed that patients receiving RIC had significantly worse OS than those receiving MAC. However, this difference was only marginally significant after adjusting for patients' baseline characteristics, while the propensity score analysis further diminished the statistical significance, indicating that there is some selection bias against RIC in these patients. Despite this, outcomes using RIC in younger patients were still acceptable, thus providing support for the use of RIC in younger patients who are deemed unsuitable for $\mathrm{MAC}^{28}$.

The two most widely used RIC regimens for AML patients are fludarabine plus busulfan (FB) and fludarabine plus melphalan (FM), with or without low-dose TBI. Several studies have evaluated the usefulness of individual RIC regimens. These analyses reveal that FM is associated with diminished relapse but higher NRM rates than FB, while OS was similar for both regimens ${ }^{29}$. Based on these results FM and FB were characterized by more potent antileukemic activity and lower toxicity, respectively. Another study focusing on FM showed that a higher dose of melphalan $\left(120-140 \mathrm{mg} / \mathrm{m}^{2}\right)$ reduced leukemia-related mortality without increasing NRM, resulting in better OS than a lower dose. The beneficial effect of a higher dose of melphalan was more evident when it was combined with low-dose TBI $(\leq 4 \mathrm{~Gy})$ for younger patients, those with non-CR, and those with good $\mathrm{PS}^{30}$.

We then went on to assess whether the addition of lowdose TBI during RIC improved the outcomes of allogeneic HCT from HLA-matched related donors. This evaluation revealed that OS, relapse, and NRM were comparable in patients conditioned with and without low-dose TBI. This suggests that the addition of low-dose TBI does not yield any significant benefits ${ }^{31}$. These observations are somewhat inconsistent with those of the aforementioned study ${ }^{30}$. Given that the majority of patients in this study received FB, the prognostic significance of adding low-dose TBI may depend on combined chemotherapy, which merits further investigation.

Data for both MAC and RIC allogenic HCT during CR collected over a $>10$-year period were analyzed and compared to evaluate the long-term outcomes of RIC allogeneic HCT. There was a median follow-up of 10.1 and 10.4 years for the RIC and MAC datasets, respectively, and this data was used to calculate the 10-year probabilities for OS, relapse, and NRM which were $36 \%$, $30 \%$, and $36 \%$ in RIC recipients and $40 \%, 26 \%$, and $36 \%$ in MAC recipients, respectively. In addition, the survival endpoints were not significantly different for RIC and MAC confirming the long-term efficacy of RIC allogeneic HCT and thus mitigating concerns over an increase in late relapse for patients treated with RIC ${ }^{32}$.

\section{Stem cell source}

Only around $40 \%$ of individuals find a matched related donor, and the remaining $60 \%$ need to find an alternative donor to receive allogeneic $\mathrm{HCT}^{33}$. The outcomes of matched related HCT, matched unrelated BMT, and UCBT performed during CR1 were compared and then used to evaluate the clinical utility of allogeneic HCT from an alternative donor. The 5-year OS probabilities for matched related HCT, matched unrelated BMT, and UCBT were $57 \%, 61 \%$, and 50\%, respectively. Multivariate analysis revealed that the risk of relapse was lower for matched unrelated BMT and UCBT, and the risks of NRM and overall mortality were higher for UCBT when compared to matched related HCT. These results suggest that a matched unrelated donor is an excellent alternative to a matched related donor, and UCB is a viable option when neither a matched related nor an unrelated donor is available $\mathrm{e}^{34}$.

It is plausible that the effect of graft type on post-transplant outcomes is time-dependent because the graft type can affect a variety of outcome events including engraftment, graft-versus-host disease (GVHD), and relapse due 
to the graft-versus-leukemia (GVL) effect, each of which occurs at different time points. Therefore, we examined the time-varying effects of graft type on post-transplant outcomes. When related PBSCT, unrelated BMT, and UCBT were compared with BMT we noted that there was an increase in the risk for NRM, and unrelated BMT and UCBT presented with a lower risk of relapse. These findings indicate that both relapse-free survival (RFS) and OS were comparable for both related and unrelated BMT but were worse for related PBSCT and UCBT than for related BMT. Some of the adverse effects of UCBT are only observed during the early phases of transplantation, while the adverse events associated with related PBSCT continued to develop even 2 years post-transplant ${ }^{35}$.

UCBT is currently actively performed in Japan. The primary reason for the widespread use of UCBT in Japan is the feasibility of finding suitable UCB units, which in turn can be explained by the relatively small body size of Japanese patients, reducing the number of cells needed for successful transplant. UCBT also has less stringent criteria for unit selection, namely, total nucleated cell (TNC) dose $\geq 2.0 \times 10^{7} / \mathrm{kg}$ and $4 / 6$ or better matching for HLA-A, -B, and -DR at the antigen level. There have been several attempts to determine whether such reductions in stringency adversely affect post-transplant outcomes with these studies finding that neither TNC dose nor the degree of HLA matching affected survival, suggesting that the less stringent criteria may be acceptable in Japanese patients. Furthermore, the less stringent criteria are likely to create more opportunities to find suitable UCB units ${ }^{36}$.

\section{Studies on autologous HCT}

Two studies were conducted to compare the outcomes of autologous HCT with those of allogeneic HCT from a matched sibling or unrelated donor for patients with AML in CR1. For both studies, the propensity score model was used to reduce the effects of confounding factors in the statistical comparison of the two nonrandomized treatment groups. These studies showed that RFS was not significantly different between autologous HCT and allogeneic HCT, and that autologous HCT could be a viable option for post-remission therapy when patients lack a suitable donor ${ }^{37,38}$.

For relapsed APL, arsenic trioxide (ATO) is the current treatment standard, and autologous HCT is generally considered the procedure of choice after CR2 has been achieved. The outcomes of patients undergoing autologous HCT during CR2 before and after the introduction of ATO in clinical practice were compared. The number of autologous HCTs during CR2 has increased considerably since the advent of ATO, and both RFS and OS have significantly improved because of the reduced incidence of post-transplant relapse. These findings corroborate the significant contribution of ATO in improving the prognosis of relapsed APL patients ${ }^{39}$.

\section{Other studies}

The treatment of relapse after allogeneic HCT remains a significant challenge, and the prognosis is usually dismal once patients have had a post-transplant relapse. Donor lymphocyte infusion (DLI) is one of the most common interventions for relapsed patients. When researchers evaluated 143 patients who received DLI for the treatment of post-transplant hematological relapse, the probabilities of OS at 1,2, and 5 years were $32 \%, 17 \%$, and $7 \%$, respectively. CR at the time of DLI, which was only applicable to $8 \%$ of the patients, was the strongest predictive factor for survival after DLI, and the majority of long-term survival after DLI was recorded for these parients ${ }^{40}$.

Over the last couple of decades, several innovations in transplantation practice, including HLA-typing technology, PBSCT, RIC, and various supportive care measures, have emerged almost simultaneously, often making it difficult to determine the individual contributions of these advancements. Patients with AML who underwent allogeneic HCT during CR1 or CR2 from a matched sibling or unrelated donor between 1994 and 2013 were analyzed in an effort to determine which elements contributed to improved clinical outcomes. A significant improvement in survival was observed in the later cohort (2007-2013) compared with the earlier cohort (1994-2006) for unrelated, but not related HCT. This improvement in unrelated HCT was solely due to a reduction in NRM, whereas the incidence of relapse did not change over time. Chronic GVHD was significantly lower in unrelated HCT during the later period. The finding that these improvements were observed exclusively in unrelated HCT suggests that advances in HLA-typing technology play a vital role in the enhancement of post-transplant survival ${ }^{41}$.

\section{Discussion}

Although AML is the most common indication for allogeneic HCT, AML itself is an infrequent disease. Moreover, only a subset of AML patients undergo HCT. The limited number of patients and the highly complex nature of the procedure often make it difficult to conduct prospective studies on HCT in patients with AML. Thus, observational studies using registry data serve as an important source of information under such circumstances.

Despite this there are some limitations to retrospective registry studies that should be considered when interpreting the results. These include selection bias, patient and 
treatment heterogeneity, and a lack of detailed data on specific areas of interest. Statistical methods such as multivariate analysis and propensity score matching analysis provide adjustments for known confounding factors, and a large number of patients may allow for accurate analysis. Nevertheless, there may be unknown or unmeasured factors that influence these analyses, skewing the results. In particular, the reasons for choosing to administer one treatment or another are not captured in a registry study, which precludes a fair comparison between different treatments. Such nonrandomized settings offer the potential for the introduction of bias, and this type of bias can theoretically be resolved using a prospective randomized study. However, retrospective analyses provide the best available evidence in the absence of any prospective randomized trials.

It is worth noting that patients who enter prospective studies need to be fit enough to meet strict predefined inclusion and exclusion criteria and thus do not necessarily represent the general patient population. Thus, registry studies can provide real-world information and their findings should be used to complement those of prospective studies, although they do not replace prospective studies by any means.

Acknowledging such potential limitations, focusing on AML in adults, and covering nearly all transplantation centers nationwide constitute the unique strengths of the registry studies conducted by the Adult AML Working Group at the JSHCT. The findings from these studies provide valuable insights into HCT in adults with AML. Although the group has produced only a small number of studies on certain topics including haploidentical transplantation and the molecular profiles of leukemia so far, the continued national surveillance of HCT will allow future evaluation of these issues, which will add much needed perspective to HCT in AML.

\section{Acknowledgements}

This work was supported in part by a grant from the Aichi Cancer Research Foundation (grant number: 20201-11).

\section{Author Contributions}

YA, TK, and MY wrote the paper.

\section{Conflicts of Interest}

The authors declare no conflict of interest. Disclosure forms provided by the authors are available here.

\section{References}

1. Atsuta Y. Introduction of Transplant Registry Unified Management Program 2(TRUMP2): scripts for TRUMP data analyses, part I (variables other than HLA-related data). Int J Hematol. 2016; 103: 3-10.

2. Yanada M, Takami A, Yamasaki S, Arai Y, Konuma T, Uchida $\mathrm{N}$, et al. Allogeneic hematopoietic cell transplantation for adults with acute myeloid leukemia conducted in Japan during the past quarter century. Ann Hematol. 2020; 99: 1351-60.

3. Yanada M, Takami A, Mizuno S, Mori J, Chou T, Usuki K, et al. Autologous hematopoietic cell transplantation for acute myeloid leukemia in adults: 25 years of experience in Japan. Int J Hematol. 2020; 111: 93-102.

4. Aoki J, Kanamori H, Tanaka M, Yamasaki S, Fukuda T, Ogawa $\mathrm{H}$, et al. Impact of age on outcomes of allogeneic hematopoietic stem cell transplantation with reduced intensity conditioning in elderly patients with acute myeloid leukemia. Am J Hematol. 2016; 91: 302-7.

5. Yamasaki S, Hirakawa A, Aoki J, Uchida N, Fukuda T, Ogawa $\mathrm{H}$, et al. Role of reduced-intensity conditioning allogeneic hematopoietic cell transplantation in older patients with de novo acute myeloid leukemia. Ann Hematol. 2017; 96: 289-97.

6. Yanada M, Konuma T, Mizuno S, Saburi M, Shinohara A, Tanaka M, et al. Predicting non-relapse mortality following allogeneic hematopoietic cell transplantation during first remission of acute myeloid leukemia. Bone Marrow Transplant. 2021; 56: 387-94.

7. Gratwohl A, Hermans J, Goldman JM, Arcese W, Carreras E, Devergie A, et al. Risk assessment for patients with chronic myeloid leukaemia before allogeneic blood or marrow transplantation. Chronic Leukemia Working Party of the European Group for Blood and Marrow Transplantation. Lancet. 1998; 352: $1087-92$.

8. Sorror ML, Maris MB, Storb R, Baron F, Sandmaier BM, Maloney DG, et al. Hematopoietic cell transplantation (HCT)specific comorbidity index: a new tool for risk assessment before allogeneic HCT. Blood. 2005; 106: 2912-9.

9. Yanada M, Mori J, Aoki J, Harada K, Mizuno S, Uchida N, et al. Effect of cytogenetic risk status on outcomes for patients with acute myeloid leukemia undergoing various types of allogeneic hematopoietic cell transplantation: an analysis of 7812 patients. Leuk Lymphoma. 2018; 59: 601-9.

10. Konuma T, Mizuno S, Kondo T, Yamaguchi H, Fukuda T, Uchida N, et al. Allogeneic hematopoietic cell transplantation in adult acute myeloid leukemia with 11q23 abnormality: a retrospective study of the Adult Acute Myeloid Leukemia Working Group of the Japan Society for Hematopoietic Cell Transplantation (JSHCT). Ann Hematol. 2018; 97: 2173-83.

11. Konuma T, Kondo T, Yamashita T, Uchida N, Fukuda T, Ozawa $\mathrm{Y}$, et al. Outcome of allogeneic hematopoietic stem cell transplantation in adult patients with acute myeloid leukemia harboring trisomy 8. Ann Hematol. 2017; 96: 469-78. 
12. Harada K, Doki N, Aoki J, Mori J, Machida S, Masuko M, et al. Outcomes after allogeneic hematopoietic stem cell transplantation in patients with acute myeloid leukemia harboring $\mathrm{t}(7 ; 11)$ (p15;p15). Haematologica. 2018; 103: e69-e72.

13. Mori J, Yanada M, Uchida N, Fukuda T, Sakura T, Hidaka M, et al. Outcomes of allogeneic hematopoietic cell transplantation in acute myeloid leukemia patients with abnormalities of the short arm of chromosome 17. Biol Blood Marrow Transplant. 2017; 23: 1398-404.

14. Harada K, Konuma T, Machida S, Mori J, Aoki J, Uchida N, et al. Risk stratification and prognosticators of acute myeloid leukemia with myelodysplasia-related changes in patients undergoing allogeneic stem cell transplantation: a retrospective study of the Adult Acute Myeloid Leukemia Working Group of the Japan Society for Hematopoietic Cell Transplantation. Biol Blood Marrow Transplant. 2019; 25: 1730-43.

15. Konuma T, Harada K, Yamasaki S, Mizuno S, Uchida N, Takahashi S, et al. Upfront allogeneic hematopoietic cell transplantation (HCT) versus remission induction chemotherapy followed by allogeneic HCT for acute myeloid leukemia with multilineage dysplasia: A propensity score matched analysis. Am J Hematol. 2019; 94: 103-10.

16. Takagi S, Masuoka K, Uchida N, Kurokawa M, Nakamae H, Imada $\mathrm{K}$, et al. Allogeneic hematopoietic cell transplantation for leukemic transformation preceded by Philadelphia chromosome-negative myeloproliferative neoplasms: a nationwide survey by the Adult Acute Myeloid Leukemia Working Group of the Japan Society for Hematopoietic Cell Transplantation. Biol Blood Marrow Transplant. 2016; 22: 2208-13.

17. Yamasaki S, Aoki J, Mori J, Harada K, Sawa M, Uchida N, et al. Effect of allogeneic stem cell transplantation in patients with minimally differentiated acute myeloid leukemia. J Hematopoietic Cell Transplant. 2019; 8: 50-9.

18. Ishiyama K, Yamaguchi T, Eto T, Ohashi K, Uchida N, Kanamori H, et al. Acute megakaryoblastic leukemia, unlike acute erythroid leukemia, predicts an unfavorable outcome after allogeneic HSCT. Leuk Res. 2016; 47: 47-53.

19. Konuma T, Kondo T, Kawata T, Iwato K, Sato Y, Mori T, et al. Hematopoietic cell transplantation for acute panmyelosis with myelofibrosis: a retrospective study in Japan. Biol Blood Marrow Transplant. 2019; 25: e23-7.

20. Mori J, Ishiyama K, Yamaguchi T, Tanaka J, Uchida N, Kobayashi T, et al. Outcomes of allogeneic hematopoietic cell transplantation in patients with biphenotypic acute leukemia. Ann Hematol. 2016; 95: 295-300.

21. Aoki J, Ishiyama K, Taniguchi S, Fukuda T, Ohashi K, Ogawa $\mathrm{H}$, et al. Outcome of allogeneic hematopoietic stem cell transplantation for acute myeloid leukemia patients with central nervous system involvement. Biol Blood Marrow Transplant. 2014; 20: 2029-33.

22. Yamasaki S, Aoki J, Mori J, Mizuno S, Uchida N, Ohashi K, et al. Better disease control before allogeneic stem cell transplantation is crucial to improve the outcomes of transplantation for acute myeloid leukemia patients with extramedullary disease.
Bone Marrow Transplant. 2020; 55: 249-52.

23. Konuma T, Yanada M, Yamasaki S, Kuwatsuka Y, Fukuda T, Kobayashi T, et al. Allogeneic haematopoietic cell transplantation for adult acute myeloid leukaemia in second remission: a retrospective study of the Adult Acute Myeloid Leukaemia Working Group of the Japan Society for Haematopoietic Cell Transplantation (JSHCT). Br J Haematol. 2018; 182: 245-50.

24. Yanada M, Mori J, Aoki J, Masuko M, Harada K, Uchida N, et al. Allogeneic hematopoietic cell transplantation for patients with a history of multiple relapses of acute myeloid leukemia. Ann Hematol. 2019; 98: 2179-86.

25. Arai Y, Takeda J, Aoki K, Kondo T, Takahashi S, Onishi Y, et al. Efficiency of high-dose cytarabine added to CY/TBI in cord blood transplantation for myeloid malignancy. Blood. 2015; 126: $415-22$.

26. Arai Y, Aoki K, Takeda J, Kondo T, Eto T, Ota S, et al. Clinical significance of high-dose cytarabine added to cyclophosphamide/total-body irradiation in bone marrow or peripheral blood stem cell transplantation for myeloid malignancy. J Hematol Oncol. 2015; 8: 102.

27. Konuma T, Kondo T, Mizuno S, Doki N, Aoki J, Fukuda T, et al. Conditioning intensity for allogeneic hematopoietic cell transplantation in acute myeloid leukemia patients with poor-prognosis cytogenetics in first complete remission. Biol Blood Marrow Transplant. 2020; 26: 463-71.

28. Yanada M, Kurosawa S, Kobayashi T, Ozawa Y, Kanamori H, Kobayashi N, et al. Reduced-intensity conditioning allogeneic hematopoietic cell transplantation for younger patients with acute myeloid leukemia: a registry-based study. Bone Marrow Transplant. 2017; 52: 818-24.

29. Yamashita T, Takami A, Uchida N, Fukuda T, Eto T, Shiratori S, et al. Reduced-intensity stem cell transplantation for acute myeloid leukemia with fludarabine-based conditioning with intravenous busulfan versus melphalan. Bone Marrow Transplant. 2020; 55: 1955-65.

30. Harada K, Yanada M, Machida S, Kanamori H, Onizuka M, Ozawa Y, et al. Prognostic impact of melphalan dose and total body irradiation use in patients with acute myeloid leukemia undergoing allogeneic stem cell transplantation with reducedintensity conditioning. Leuk Lymphoma. 2019; 60: 1493-502.

31. Aoki J, Seo S, Kanamori H, Tanaka M, Fukuda T, Onizuka M, et al. Impact of low-dose TBI on outcomes of reduced intensity conditioning allogeneic hematopoietic stem cell transplantation for AML. Bone Marrow Transplant. 2016; 51: 604-6.

32. Yanada M, Fukuda T, Tanaka M, Ota S, Toya T, Mori T, et al. Long-term results of reduced-intensity conditioning allogeneic hematopoietic cell transplantation for older patients with acute myeloid leukemia: a retrospective analysis of 10-year followup data. Bone Marrow Transplant. 2020; 55: 2008-16.

33. Yanada M, Kurosawa S, Yamaguchi T, Uchida N, Miyawaki S, Kanamori H, et al. Effect of related donor availability on outcome of AML in the context of related and unrelated hematopoietic cell transplantation. Bone Marrow Transplant. 2013; 48: $390-5$. 
34. Yano S, Yokoyama H, Yanada M, Mori J, Aoki J, Ohashi K, et al. Role of alternative donor allogeneic hematopoietic stem cell transplantation in patients with intermediate- or poor-risk acute myeloid leukemia in first complete remission. Bone Marrow Transplant. 2019; 54: 2004-12.

35. Yanada M, Konuma T, Yamasaki S, Kuwatsuka Y, Masuko M, Tanaka M, et al. Time-varying effects of graft type on outcomes for patients with acute myeloid leukemia undergoing allogeneic hematopoietic cell transplantation. Biol Blood Marrow Transplant. 2020; 26: 307-15.

36. Yanada M, Konuma T, Kuwatsuka Y, Kondo T, Kawata T, Takahashi $\mathrm{S}$, et al. Unit selection for umbilical cord blood transplantation for adults with acute myeloid leukemia in complete remission: a Japanese experience. Bone Marrow Transplant. 2019; 54: 1789-98.

37. Mizutani M, Hara M, Fujita H, Aoki J, Kanamori H, Ohashi K, et al. Comparable outcomes between autologous and allogeneic transplant for adult acute myeloid leukemia in first CR. Bone Marrow Transplant. 2016; 51: 645-53.

38. Mizutani M, Takami A, Hara M, Mizuno S, Yanada M, Chou T, et al. Comparison of autologous and unrelated transplants for cytogenetically normal acute myelogenous leukemia. Biol Blood Marrow Transplant. 2017; 23: 1447-54.

39. Yanada M, Yano S, Kanamori H, Gotoh M, Emi N, Watakabe K, et al. Autologous hematopoietic cell transplantation for acute promyelocytic leukemia in second complete remission: outcomes before and after the introduction of arsenic trioxide. Leuk Lymphoma. 2017; 58: 1061-7.

40. Takami A, Yano S, Yokoyama H, Kuwatsuka Y, Yamaguchi T, Kanda Y, et al. Donor lymphocyte infusion for the treatment of relapsed acute myeloid leukemia after allogeneic hematopoietic stem cell transplantation: a retrospective analysis by the Adult Acute Myeloid Leukemia Working Group of the Japan Society for Hematopoietic Cell Transplantation. Biol Blood Marrow Transplant. 2014; 20: 1785-90.

41. Yanada M, Masuko M, Mori J, Aoki J, Mizuno S, Fukuda T, et al. Patients with acute myeloid leukemia undergoing allogeneic hematopoietic cell transplantation: trends in survival during the past two decades. Bone Marrow Transplant. 2019; 54: 578-86.

https://doi.org/10.31547/bct-2020-023

Copyright (C) 2021 APBMT. All Rights Reserved. 